\title{
Counting Single Redox Turnovers: Fluorogenic Antioxidant Conversion and Mass Transport Visualization via Single Molecule Spectroelectrochemistry
}

Robert Godin, and Gonzalo Cosa*

Department of Chemistry and Center for Self-Assembled Chemical Structures (CSACS/CRMAA), McGill University, 801 Sherbrooke Street West, Montreal, QC, H3A 0B8, Canada.

*Email: gonzalo.cosa@mcgill.ca

\section{Contents}

Detailed SMS-EC Experimental Methods .................................................................. 2

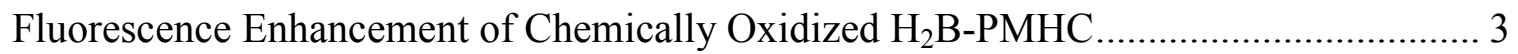

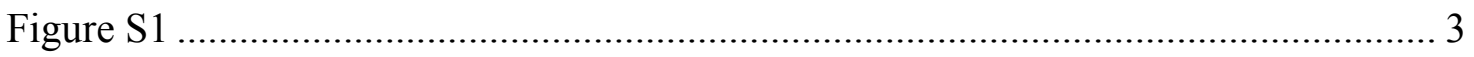

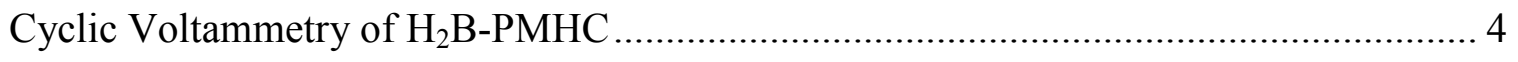

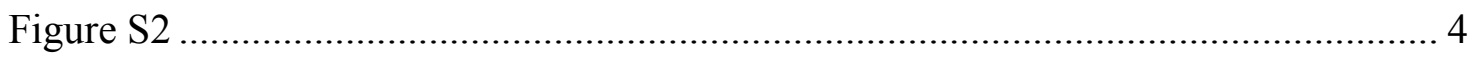

Determination of Concentration Profile by Fluorescence Burst Analysis ........................ 5

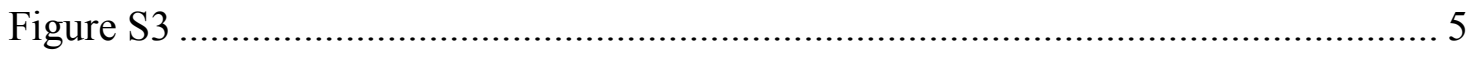

Fluorescence Correlation Spectroscopy (FCS) Analysis ............................................ 6

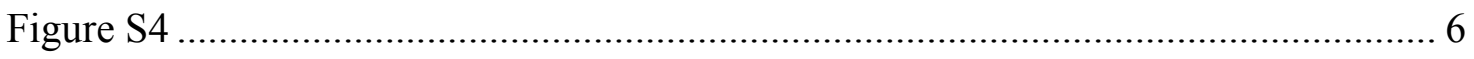

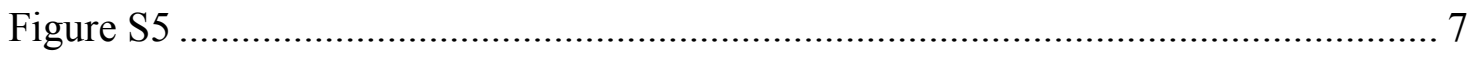

Effect of $\mathrm{O}_{2}$ under Electroreductive Conditions ................................................... 8

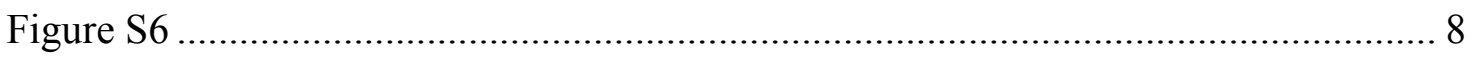

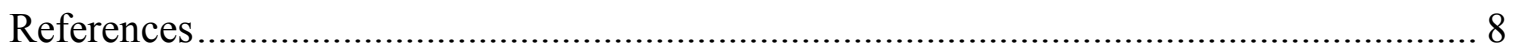




\section{Detailed SMS-EC Experimental Methods}

Materials. Anhydrous acetonitrile, cerium(IV) ammonium nitrate, and tetrabutylammonium perchlorate were purchased from Sigma-Aldrich and used without further purification. $\mathrm{H}_{2} \mathrm{~B}-\mathrm{PMHC}$ was synthesized according to literature procedures. ${ }^{1}$

The ITO coverslips (Evaporated Coatings Inc., $50 \Omega / \square$; cleaned by 20 minutes sequential sonication in acetone, trichloroethylene and methanol) were platinized to improve the catalytic activity of the electrode. Platinum was deposited by spin coating a drop of $\sim 20 \mu \mathrm{L}$ of platinum precursor solution (Plastisol T, Solaronix). The coverslips were then sintered at $450{ }^{\circ} \mathrm{C}$ for 30 minutes. After cooling to allow for handling, a glass tube was glued with epoxy (Hardman ${ }^{\circledR}$, McMaster-Carr) on top of the coverslip to form the sample chamber. The epoxy glue was cured by placing the samples in a $110{ }^{\circ} \mathrm{C}$ oven for $\sim 30$ minutes. The sample chamber was filled with the desired analyte solution and sealed by a septum pierced with the reference and counter electrodes. When oxygen-free conditions were required, argon was bubbled through the solution for 1 hour in order to deaerate the solution, and an argon-filled balloon was fixed on the sample during acquisition. The electrochemical cell was then mounted on the microscope (IX-71, Olympus). The electrochemical potential was controlled by a CHI600D electrochemical analyzer. The AgCl-coated Ag wire quasireference electrode used was calibrated with the ferrocene/ferrocenium redox couple using $\mathrm{E}_{\mathrm{Fc}+\mathrm{Fc}}^{0}=+0.64 \mathrm{~V}$ vs. NHE. All potentials reported are referenced to $\mathrm{NHE}$.

The laser excitation was provided by a supercontinuum laser (WhiteLase SC-400-4, Fianium, Beverly, MA). A center excitation wavelength of $498.2 \mathrm{~nm}$ was spectrally separated from the broadband emission by a computer-controlled acousto-optical tunable filter (AOTF, Fianium). A Z488rdc dichroic mirror (Chroma, Rockingham, VT) projected the pulsed laser excitation (20 MHz repetition rate, $24 \mu \mathrm{W}$ out of the objective) towards the water-immersion objective (UPLSAPO 60XW, N.A. = 1.20, Olympus). The confocal observation volume was focused between 20 and $80 \mu \mathrm{m}$ above the electrode surface, as controlled by a piezoelectric objective scanner (PIFOC P-721 controlled by an E710, Physik Instrumente (PI), Auburn, MA). The emission in a 500-580 nm spectral window (ET540/80 bandpass filter, Chroma) was collected by the same objective, passed through a $100 \mu \mathrm{m}$ diameter pinhole positioned at the image plane, and split onto two avalanche photodiode detectors (APDs; SPCM-AQR-14, Perkin Elmer Optoelectronics, Vaudreuil, QC) with a 50:50 non-polarizing beamsplitter in pseudocross-correlation format. ${ }^{2}$ This configuration was used to observe microsecond dynamics that are obscured by detector afterpulsing in a single-detector setup. The data acquisition, signal from the detectors and the piezo scanner were controlled and analyzed by software from PicoQuant (SymPhoTime). 


\section{Fluorescence Enhancement of Chemically Oxidized $\mathrm{H}_{2}$ B-PMHC}

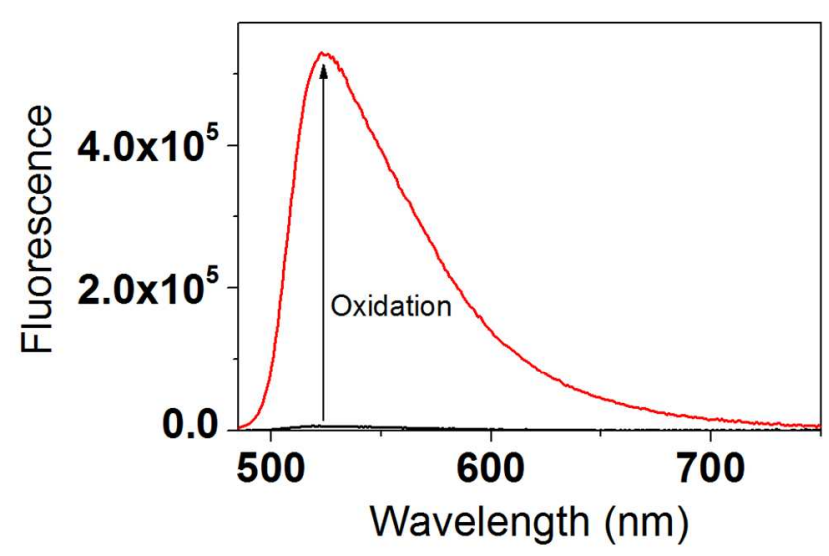

Figure S1. Fluorescence enhancement observed in acetonitrile solutions upon chemical oxidation of $\mathrm{H}_{2} \mathrm{~B}$-PMHC $(1 \mu \mathrm{M})$ by 2 eq. of cerium(IV) ammonium nitrate. An 83-fold fluorescence enhancement is calculated by integrating the intensities between $485-675 \mathrm{~nm}$ following emission correction for background. $\lambda_{\mathrm{ex}}=480 \mathrm{~nm}$. 


\section{Cyclic Voltammetry of $\mathrm{H}_{2} \mathrm{~B}-\mathrm{PMHC}$}

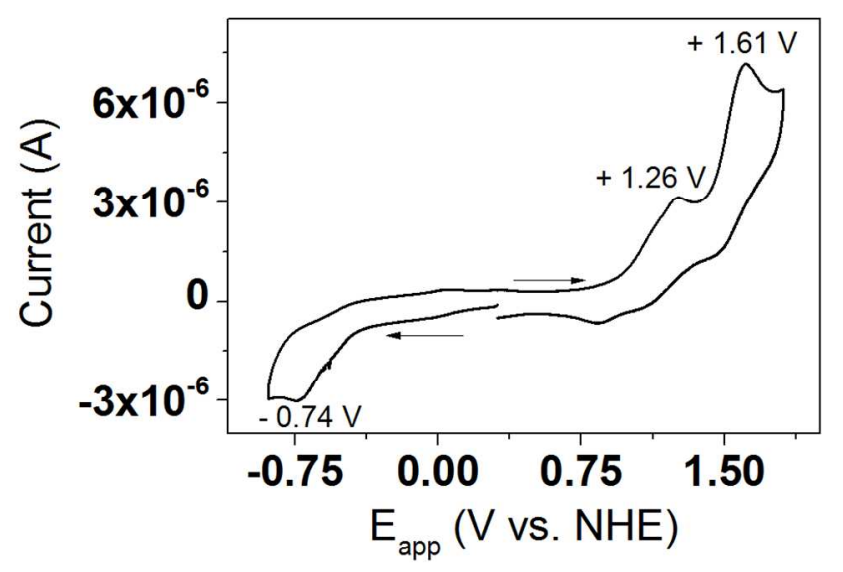

Figure S2. Cyclic voltammogram of $\mathrm{H}_{2} \mathrm{~B}-\mathrm{PMHC}(54 \mu \mathrm{M})$ in argon-purged $0.1 \mathrm{M} \mathrm{Bu}_{4} \mathrm{NClO}_{4}$ acetonitrile solution. The experiment was run in the SMS-EC cell: Pt/ITO served as the working electrode, AgCl-coated Ag wire as the quasireference electrode and a Pt wire as the counter electrode. The anodic peaks at $1.26 \mathrm{~V}$ and $1.61 \mathrm{~V}$ are assigned to the oxidation of the chromanol and BODIPY core, respectively. The cathodic peak at $-0.74 \mathrm{~V}$ is assigned to the reduction of the BODIPY core, in accordance with reduction potentials values for similar BODIPY dyes $(\sim-0.8$ $\mathrm{V}){ }^{3}$ The potential sweep rate was $0.1 \mathrm{~V} / \mathrm{s}$. 


\section{Determination of Concentration Profile by Fluorescence Burst Analysis}

A

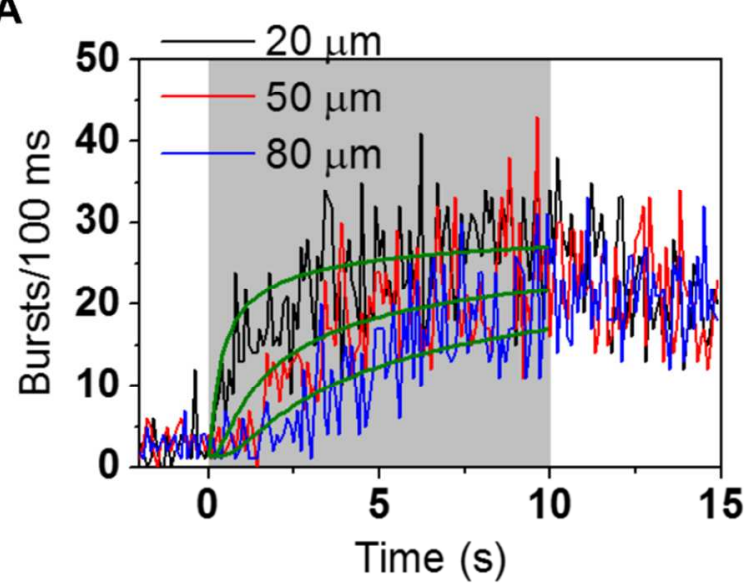

B

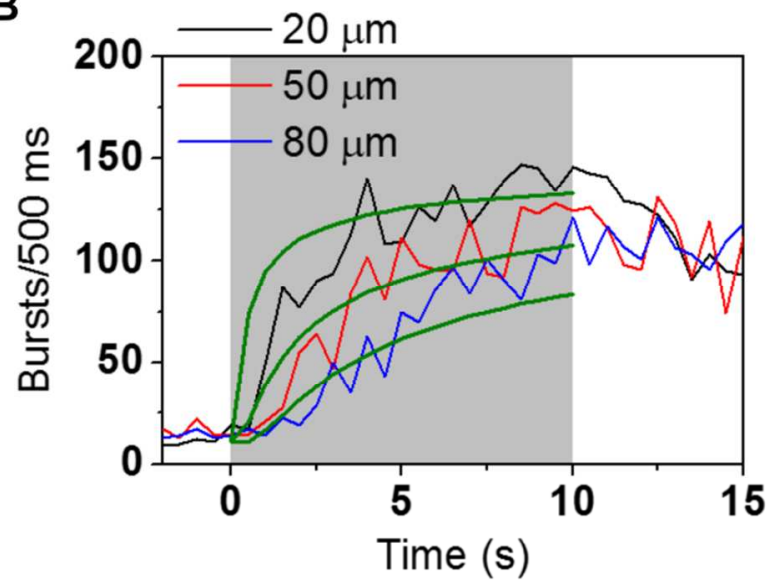

Figure S3. SMS-EC determination of the concentration profile of electrochemically-generated $\mathrm{H}_{2} \mathrm{~B}-\mathrm{PMHC}_{\mathrm{ox}}$ by a fluorescence burst analysis. The number of fluorescent bursts observed are summed over $100 \mathrm{~ms}$ (A) or $500 \mathrm{~ms}$ (B) to generate the fluorescence burst frequencies trajectories. A fluorescent burst was counted each time the number of counts (photons) in a $50 \mu \mathrm{s}$ time bine was greater than 4 . The $\mathrm{H}_{2} \mathrm{~B}-\mathrm{PMHC}$ solutions were under the influence of the bias shown in Figure 3A of the main text. The confocal observation volumes were located $20 \mu \mathrm{m}$ (black), $50 \mu \mathrm{m}$ (red) and $80 \mu \mathrm{m}$ (blue) above the electrode surface. The time axis was set to $0 \mathrm{~s}$ at the start of the $+1.26 \mathrm{~V}$ pulse (highlighted in gray). Green lines represent the best fit result of the model described by Equation 2 of the main text. Similar results to the fluorescence intensity analysis were obtained. Explicitly, global fitting yielded $D=8.1 \times 10^{-10} \mathrm{~m}^{2} \mathrm{~s}^{-1}$ and $\mathrm{D}=7.6 \times 10^{-10}$ $\mathrm{m}^{2} \mathrm{~s}^{-1}$ for $\mathrm{A}$ ) and $\left.\mathrm{B}\right)$, respectively. 


\section{Fluorescence Correlation Spectroscopy (FCS) Analysis}

FCS traces were software-generated using SymPhoTime (PicoQuant) and analysed using the open-sourced program PyCorrFit. ${ }^{4}$ A 3D Gaussian model with 1 diffusional and 1 triplet terms,

$$
G(\tau)=\frac{1}{\langle N\rangle}\left(1+\frac{\tau}{\tau_{D}}\right)^{-1}\left(1+\frac{\tau}{\kappa^{2} \tau_{D}}\right)^{-1 / 2}\left[1+\frac{T}{1-T} \exp \left(-\tau / \tau_{T}\right)\right]
$$

gave satisfactory fits. $\mathrm{G}(\tau)$ is the amplitude of the autocorrelation function, $<\mathrm{N}>$ is the average number of molecules in the observation volume, $\tau$ is the correlation lag time, $\tau_{\mathrm{D}}$ is the translational diffusional time, $\kappa$ is the structure parameter, $\mathrm{T}$ is the triplet fraction and $\tau_{\mathrm{T}}$ is the characteristic triplet time. The structure parameter, the ratio of the long axis $\left(\omega_{z}\right)$ to the short axis $\left(\omega_{x y}\right)$ of the observation volume $\left(\kappa=\omega_{z} / \omega_{x y}\right)$ were obtained from fits of traces for $\mathrm{H}_{2} \mathrm{~B}-\mathrm{PMHC} \mathrm{ox}_{\mathrm{o}}$ had values $\sim 5$ and were fixed when fitting trajectories of $\mathrm{H}_{2} \mathrm{~B}-\mathrm{PMHC}$ under electrooxidative conditions. $\quad<\mathrm{N}>$ at different points of a trajectory could then be determined from fits. For determination of the diffusion coefficient, the confocal volume was calibrated using the known concentration $(10 \mathrm{nM})$ of a fluorescent dye $\left(\mathrm{H}_{2} \mathrm{~B}-\mathrm{PMHC}-\mathrm{OMe}\right)$ with similar structure to $\mathrm{H}_{2} \mathrm{~B}$ $\mathrm{PMHC}_{(\mathrm{ox})}$. This calibration was then used to determine the diffusion coefficient (D) from fitting a trace acquired in the presence of $500 \mathrm{pM} \mathrm{H}_{2} \mathrm{~B}-\mathrm{PMHC}$ ox according to $D=\omega_{x y}^{2} / 4 \tau_{D}$.

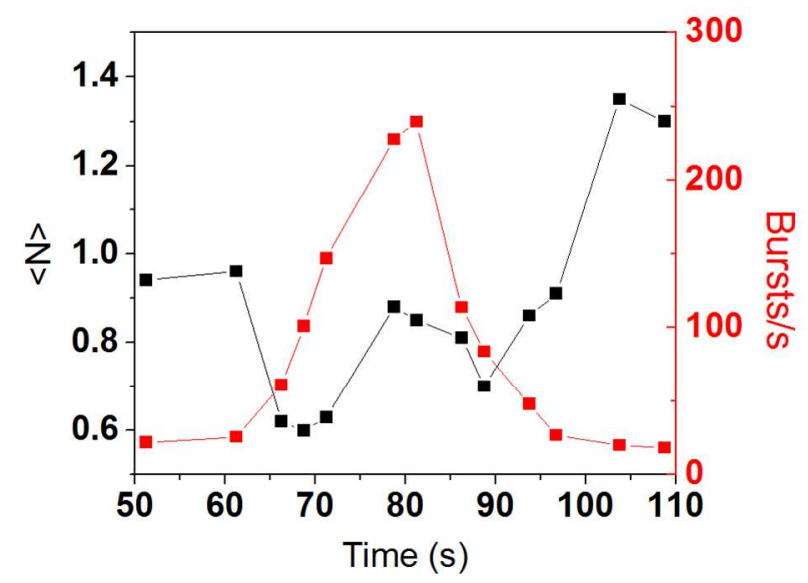

Figure S4. Time evolution of the fluorescence bursts and average number of molecules in the observation volume for the trajectory shown in Figure 2 of main text. FCS traces were generated from $2.5 \mathrm{~s}$ long segments centered at the time points shown. $<\mathrm{N}>$ was then determined from these traces by fitting. The $<\mathrm{N}>$ values trace the fluorescent bursts frequency near the high intensity peak, indicating a correlation between the number of $\mathrm{H}_{2} \mathrm{~B}-\mathrm{PMHC}_{\mathrm{ox}}$ and burst frequency. Deviations between $\langle\mathrm{N}\rangle$ and the burst frequency occur in the low fluorescence intensity regions due to interference from fluorescent impurities in determining $<\mathrm{N}>$. For multiple species, the initial amplitude is given by $G(0)=\frac{1}{\langle N\rangle}=\frac{\sum N_{i} B_{i}^{2}}{\left(\sum N_{i} B_{i}\right)^{2}}$ where $\mathrm{B}$ is the brightness of the $i$ th species. 


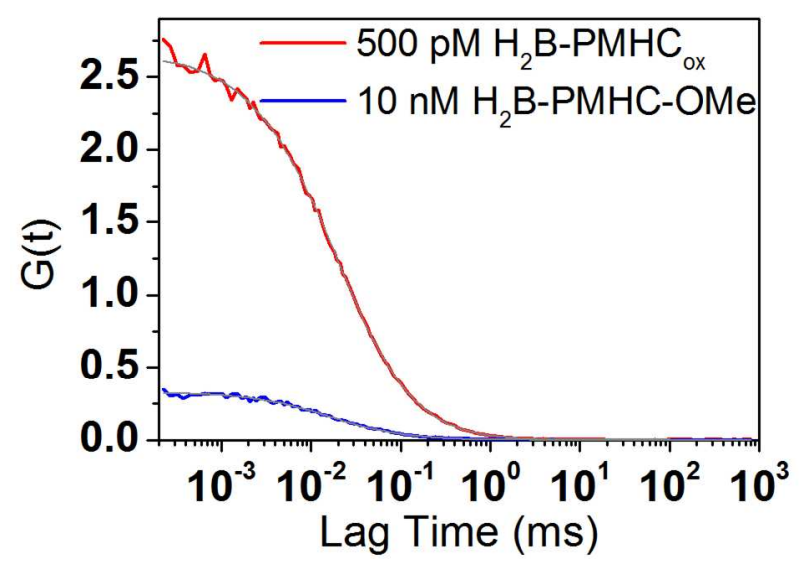

Figure S5. FCS traces of oxidized $\mathrm{H}_{2} \mathrm{~B}-\mathrm{PMHC}$ (red) and a fluorescent analogue (blue) acquired at $80 \mu \mathrm{m}$ from the electrode surface. Fits to the model used are shown in gray. The experimental setup was calibrated from the known $10 \mathrm{nM}$ concentration of $\mathrm{H}_{2} \mathrm{~B}-\mathrm{PMHC}-\mathrm{OMe}$. The high concentration mitigated effects of impurities in determining $<\mathrm{N}>$. Fitting of the $500 \mathrm{pM} \mathrm{H}_{2} \mathrm{~B}$ $\mathrm{PMHC}_{\mathrm{ox}}$ was then used to extract $D=8.5 \times 10^{-10} \mathrm{~m}^{2} \mathrm{~s}^{-1}$. 


\section{Effect of $\mathrm{O}_{2}$ under Electroreductive Conditions}

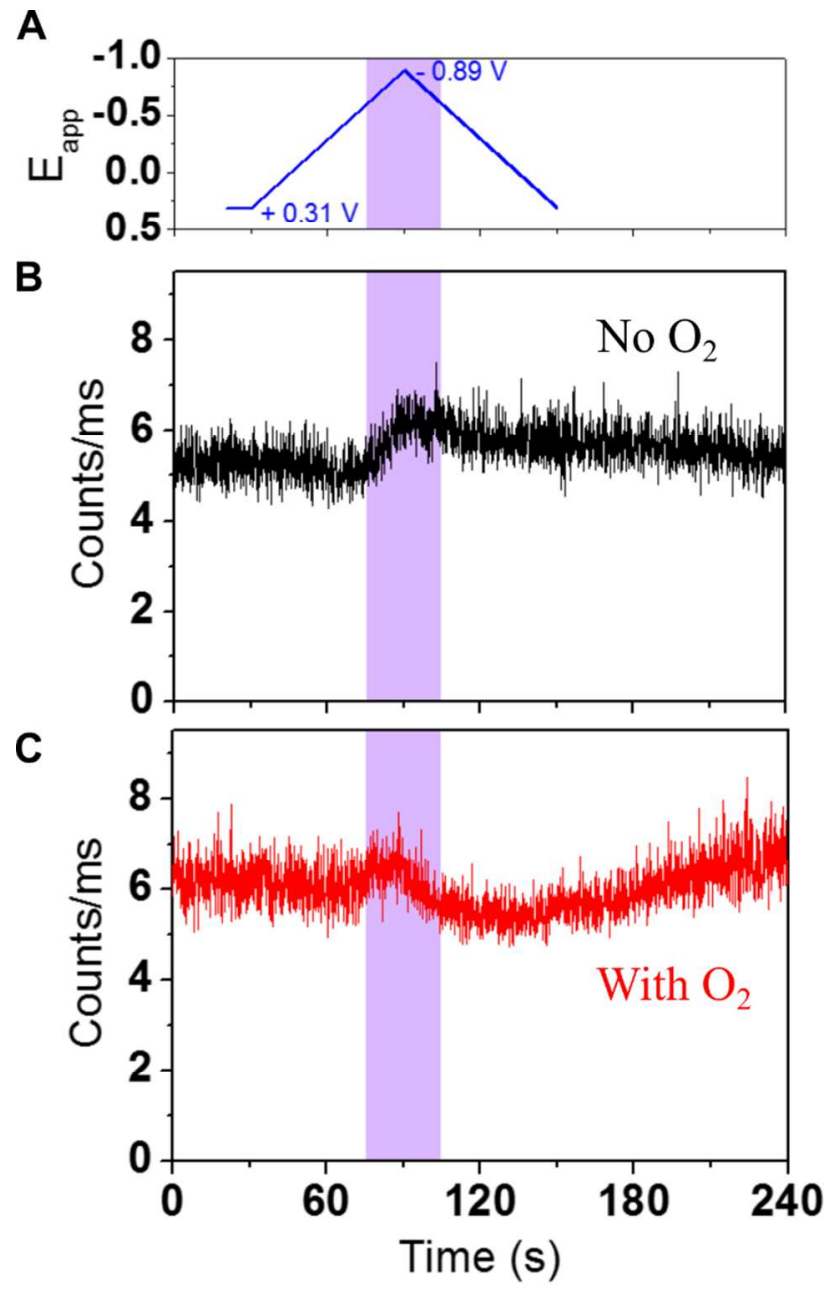

Figure S6. Effect of the presence of oxygen under reductive conditions. A) Applied electrochemical potential. The potential scan rate was $20 \mathrm{mV} / \mathrm{s}$. B and C) Fluorescence trajectories of $\mathrm{H}_{2} \mathrm{~B}-\mathrm{PMHC}$ under influence of the bias shown in A). B) Argon-purged conditions. C) Air-equilibrated conditions. Times where $\mathrm{E}_{\mathrm{app}} \leq-0.6 \mathrm{~V}$ are highlighted in purple. $\mathrm{H}_{2} \mathrm{~B}-\mathrm{PMHC}$ concentration was $0.5 \mathrm{nM}$.

\section{References}

(1) Krumova, K.; Friedland, S.; Cosa, G. J. Am. Chem. Soc. 2012, 134, 10102.

(2) Yu, J.; Lammi, R.; Gesquiere, A. J.; Barbara, P. F. J. Phys. Chem. B 2005, 109, 10025.

(3) (a) Krumova, K.; Cosa, G. J. Am. Chem. Soc. 2010, 132, 17560.(b) Lincoln, R.; Greene, L.

E.; Krumova, K.; Ding, Z.; Cosa, G. J. Phys. Chem. A 2014, 118, 10622.

(4) Müller, P.; Schwille, P.; Weidemann, T. Bioinformatics 2014, 30, 2532. 